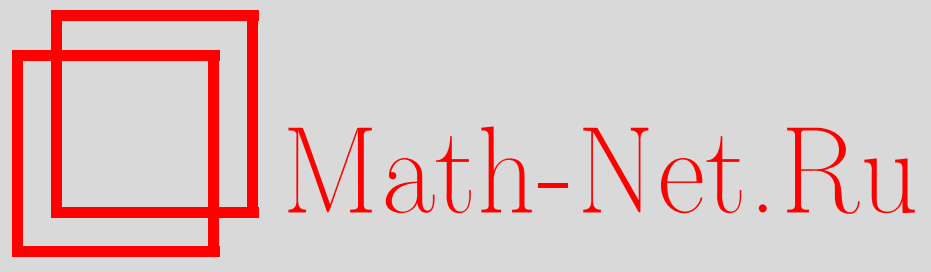

A. P. Миротин, Об одном классе операторно-монотонных функций нескольких переменных, Матем. заметки, 2013, том 94, выпуск 1, 154-156

DOI: https://doi.org/10.4213/mzm9076

Использование Общероссийского математического портала Math-Net.Ru подразумевает, что вы прочитали и согласны с пользовательским соглашением http://www . mathnet.ru/rus/agreement

Параметры загрузки:

IP : 54.81 .137 .203

26 апреля 2023 г., 13:21:21

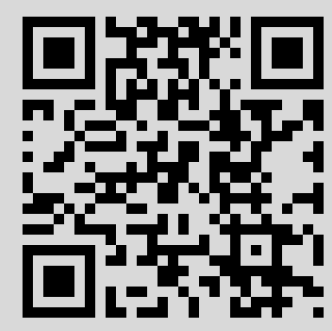




\section{Об одном классе}

\section{операторно-монотонных функций нескольких переменных}

\section{А. Р. Миротин}

Классическая теорема Лёвнера [1]-[3] (см. также [4; замечания к §Х.2]) утверждает, что непрерывная функция $f:(0,+\infty) \rightarrow \mathbb{R}$ обладает свойством $f(A) \leqslant f(B)$ для всех пар ограниченных самосопряженных операторов $A, B$ в гильбертовом пространстве $H$ таких, что $0<A \leqslant B$, тогда и только тогда, когда $f$ аналитически продолжается в область $\mathbb{C} \backslash(-\infty, 0]$ и это продолжение отображает открытую верхнюю полуплоскость в свое замыкание. Такие функции называют операторно-монотонными функциями (ОМФ) одного переменного. Они находят важные применения в анализе, математической физике и теории электрических сетей (см. [3], [5] и библиографию там). Заметим, что, в силу принципа максимума, ОМФ одного переменного либо отображает открытую верхнюю полуплоскость в себя, либо является вещественной постоянной.

ОМФ одного переменного, отображающие луч $(0,+\infty)$ в себя, называются неотрицательными ОМФ одного переменного. Известно ([5], [6; лемма 5.2]), что неотрицательные ОМФ одного переменного имеют в точности вид

$$
f(z)=a z+b+\int_{0}^{\infty} \frac{z}{z+t} d \mu(t), \quad z \in \mathbb{C} \backslash(-\infty, 0),
$$

где $a, b \geqslant 0$, а $\mu$ - положительная мера на $\mathbb{R}_{+}$. Отсюда, в частности, следует, что непостоянная неотрицательная ОМФ одного переменного отображает и открытую нижнюю полуплоскость в себя.

Функции, голоморфные в области $\mathbb{C} \backslash[0,+\infty)$ и отображающие открытую верхнюю полуплоскость в свое замыкание, а луч $(-\infty, 0)$ - в себя, будем называть неположительными $О М \Phi$ одного переменного. Из сказанного выше ясно, что $g(z)$ будет неположительной ОМФ одного переменного тогда и только тогда, когда $-g(-z)$ является неотрицательной ОМФ одного переменного. При этом неравенство $g(C) \leqslant g(D)$ выполняется для всех пар ограниченных самосопряженных операторов $C, D$ в гильбертовом пространстве $H$ таких, что $C \leqslant D<0$.

Поскольку функция $z \mapsto-z^{-1}$ является операторно-монотонной (доказательство этого факта, независимое от теоремы Лёвнера, см. в [7; гл. II, §47]), имеет место

Лемма 1. Пусть $A, B$ - ограниченные самосопряженные операторы в гильбертовом пространстве $H, R(\lambda, A)=(\lambda I-A)^{-1}$.

1) Если $A \leqslant B<0$, то для любого числа $\lambda>0$ справедливы неравенства для резольвент $0<R(\lambda, A) \leqslant R(\lambda, B)$.

2) Если $0<R(\lambda, A) \leqslant R(\lambda, B)$ для некоторого $\lambda>0$, mо $A \leqslant B$.

Для формулировки основного результата введем многомерный аналог класса неположительных ОМФ одного переменного. Напомним [8], что функция $\psi$ на $(-\infty ; 0]^{n}$ принадлежит классу $\mathcal{T}_{n}$, если она неположительна, и все ее частные производные первого порядка абсолютно монотонны (неотрицательная непрерывная функция на $(-\infty ; 0]^{n}$ называется абсолютно монотонной, если она принадлежит классу $C^{\infty}(-\infty ; 0)^{n}$ и все ее частные производные неотрицательны на $(-\infty ; 0)^{n}$, см., например, $[9 ;$ дополнения и задачи к гл. 5, п. 16]). Функции класса $\mathcal{T}_{n}$ будем называть неположителъными функциями Бернштейна $n$ переменных. Известно [8], [10], что неположительная функция Бернштейна $n$

Работа выполнена при поддержке Государственной программы фундаментальных исследований Республики Беларусь (грант № 20111164).

DOI: $10.4213 / \operatorname{mzm} 9076$ 
переменных имеет вид

$$
\psi(s)=c_{0}+c_{1} \cdot s+\int_{\mathbb{R}_{+}^{n} \backslash\{0\}}\left(e^{s \cdot u}-1\right) d \mu(u),
$$

где $c_{0}=\psi(-0) \leqslant 0, c_{1}=\nabla \psi(-\infty) \in \mathbb{R}_{+}^{n}$, а положительная мера $\mu$ на $\mathbb{R}_{+}^{n} \backslash\{0\}$ определяется по функции $\psi$ однозначно. Кроме того, $\psi$ продолжается по формуле $(2)($ при $s=z)$ до функции, непрерывной в замкнутой области $\left\{\operatorname{Re} z_{j} \leqslant 0\right\} \subset \mathbb{C}^{n}$ и голоморфной внутри этой области. При $n=1$ из $(2)$ также следует, что $\psi$ либо отображает луч $(-\infty ; 0)$ в себя, либо равна нулю.

ОпредЕЛЕниЕ 1. Скажем, что неположительная функция Бернштейна $n$ переменных $\psi$ принадлежит классу $\mathcal{R}_{n}^{-}$, если существует такие числа $\lambda>0, \gamma \geqslant 0$ и положительная мера $\tau$ на $\mathbb{R}_{+}^{n}$, что при всех $z \in \mathbb{C}^{n}$ с $\operatorname{Re} z_{j}>0, j=1, \ldots, n$, выполняется равенство

$$
\frac{1}{\lambda-\psi(-z)}=\gamma+S \tau(z)
$$

где

$$
S \tau(z)=\int_{\mathbb{R}_{+}^{n}} \frac{d \tau(\xi)}{\left(\xi_{1}+z_{1}\right) \ldots\left(\xi_{n}+z_{n}\right)}
$$

суть $n$-мерное преобразование Стилтьеса меры $\tau$.

ЗАмЕчАниЕ. Класс $\mathcal{R}_{1}^{-}$совпадает с классом неположительных ОМФ одного переменного. В самом деле, пусть функция $\psi \in \mathcal{R}_{1}^{-}$не есть тождественный нуль. Тогда она отображает луч $(-\infty ; 0)$ в себя. Кроме того, из $(3)$ при $n=1$ следует, что функция $F(z)=$ $1 /(\lambda-\psi(-z))$ принадлежит классу $\mathcal{S}^{-}$в смысле $[11 ;$ гл. $\mathrm{V}, \S 3]$. А тогда функция $\psi(\zeta)=$ $\lambda-1 / F(-\zeta)$ голоморфна в области $\mathbb{C} \backslash[0,+\infty)$ и отображает открытую верхнюю полуплоскость в себя. Таким образом, $\psi$ есть неположительная ОМФ Обратно, если $g$ есть неположительная ОМФ, то $-g(-s)$ есть неотрицательная ОМФ, а потому $g$ имеет вид

$$
g(s)=a s-b+\int_{0}^{\infty} \frac{s}{t-s} d \mu(t), \quad s \in(-\infty, 0) .
$$

Подставляя в правую часть $s /(t-s)=\int_{0}^{\infty}\left(e^{s u}-1\right) t e^{-t u} d u$ и применяя теорему Фубини, получаем, что $g$ имеет вид $(2)$, т.е. принадлежит $\mathcal{T}_{1}$. Наконец, при $\lambda>0$ функция $1 /(\lambda-$ $g(-z))$ принадлежит $\mathcal{S}^{-}$, а потому имеет вид $(3)[11 ;$ гл. $\mathrm{V}, \S 3]$.

Внутренняя характеристика класса $\mathcal{R}_{n}^{-}$при $n>1$ могла бы быть получена аналогичным образом, если бы была известна внутренняя характеристика класса функций $n$ переменных, представимых в виде $n$-мерного преобразования Стилтьеса положительных мер.

Пусть $A=\left(A_{1}, \ldots, A_{n}\right)$ - набор ограниченных попарно коммутирующих неположительных операторов в $H$. Поскольку любая неположительная функция Бернштейна $n$ переменных $\psi$ непрерывна в области $(-\infty ; 0]^{n}$, для нее, как известно, можно определить оператор $\psi(A)$ следующим образом:

$$
\psi(A)=\int \ldots \int_{\Delta} \psi\left(s_{1}, \ldots, s_{n}\right) E\left(d s_{1} \ldots d s_{n}\right),
$$

где $\Delta-$ замкнутый брус в $(-\infty ; 0]^{n}$, содержащий $\sigma\left(A_{1}\right) \times \cdots \times \sigma\left(A_{n}\right)$, а $E-$ совместное разложение единицы набора $A$ ([12; п. 111]). 
Целью данного сообщения является

Теорема 1. Пусть $\psi$ принадлежит $\mathcal{R}_{n}^{-}$. Тогда для любых наборов $A=\left(A_{1}, \ldots, A_{n}\right) u$ $A^{\prime}=\left(A_{1}^{\prime}, \ldots, A_{n}^{\prime}\right)$ ограниченных попарно коммутирующих неположительных операторов в $Н$ неравенства $A_{j} \leqslant A_{j}^{\prime}, j=1, \ldots, n$, влекут неравенство $\psi(A) \leqslant \psi\left(A^{\prime}\right)$.

Теорема 1 будет вытекать из следующего утверждения, в случае операторов обобщающего теорему 5 из [13], где рассмотрен случай $\gamma=0$.

Теорема 2. Пусть $\psi$ принадлежит $\mathcal{R}_{n}^{-}$. Тогда для любого набора $A=\left(A_{1}, \ldots, A_{n}\right)$ ограниченных попарно коммутирующих неположительных операторов в $H$ справедливо равенство

$$
R(\lambda, \psi(A))=\gamma I+\int_{\mathbb{R}_{+}^{n}} \prod_{j=1}^{n} R\left(\xi_{j}, A_{j}\right) d \tau(\xi),
$$

где числа $\lambda>0, \gamma \geqslant 0$ и мера $\tau \geqslant 0$ удовлетворлют равенству (3).

ДокАзАтЕльство. Пусть функция $\psi$ имеет интегральное представление (2). Тогда в силу теоремы Фубини

$$
\begin{aligned}
\psi(A) & =\int_{\Delta} \psi(s) E(d s)=\int_{\Delta}\left(c_{0}+c_{1} \cdot s+\int_{\mathbb{R}_{+}^{n} \backslash\{0\}}\left(e^{s \cdot u}-1\right) d \mu(u)\right) E(d s) \\
& =c_{0} I+c_{1} \cdot A+\int_{\mathbb{R}_{+}^{n} \backslash\{0\}}\left(e^{u \cdot A}-I\right) d \mu(u),
\end{aligned}
$$

где положено $r \cdot A=\sum_{j} r_{j} A_{j}$ при $r \in \mathbb{R}^{n}$. Следовательно, оператор $\psi(A)$ совпадает со значением функции $\psi$ на наборе $A$ в смысле [8]. Оставшаяся часть доказательства теоремы 2 теперь лишь немногим отличается от доказательства теоремы 5 в [13], так как операторы $A_{j}$ являются диссипативными элементами алгебры ограниченных операторов в $H$.

ДОКАЗАТЕЛЬСТВо тЕОРЕМЫ 1 получается простой комбинацией леммы 1 и теоремы 2. В самом деле, так как при всех $\xi_{j} \geqslant 0$ по лемме имеем $0<R\left(\xi_{j}, A_{j}\right) \leqslant R\left(\xi_{j}, A_{j}^{\prime}\right), j=1, \ldots, n$, то и $0<\prod_{j=1}^{n} R\left(\xi_{j}, A_{j}\right) \leqslant \prod_{j=1}^{n} R\left(\xi_{j}, A_{j}^{\prime}\right)$. Тогда $0<R(\lambda, \psi(A)) \leqslant R\left(\lambda, \psi\left(A^{\prime}\right)\right)$ при некотором $\lambda>0$ по теореме 2 , и осталось еще раз применить лемму.

Автор благодарит рецензента за замечания, способствовавшие улучшению изложения.

\section{СПИСОК ЦИТИРОВАННОЙ ЛИТЕРАТУРЫ}

[1] K. Löwner, Math. Z., 38 (1934), 177-216. [2] C. Loewner, Bull. Amer. Math. Soc., 56 (1950), 308-319. [3] J. Bendat, S. Sherman, Trans. Amer. Math. Soc., 79 (1955), 58-71. [4] М. Рид, Б. Саймон, Методы современной математической физики. Т. 2. Гармонический анализ. Самосопряженность, Мир, М., 1978. [5] M. Rosenblum, J. Rovnyak, Hardy Classes and Operator Theory, Oxford Math. Monogr., Oxford Univ. Press, Oxford, 1985. [6] C. Berg, K. Boyadzhiev, R. deLaubenfels, J. Austral. Math. Soc. Ser. A, 55:2 (1993), 246-269. [7] Э. Беккенбах, Р. Беллман, Неравенства, Мир, М., 1965. [8] А.Р. Миротин, Матем. заметки, 61:4 (1997), 630-633. [9] Н. И. Ахиезер, Классическая проблема моментов и некоторые вопросы анализа, связанные с нею, Физматгиз, М., 1961. [10] А. Р. Миротин, Алгебра и анализ, 11:2 (1999), 142-169. [11] М. Г. Крейн, А. А. Нудельман, Проблема моментов Маркова и экстремальные задачи. Идеи и проблемы П. Л. Чебышева и А. А. Маркова и их дальнейшее развитие, Наука, М., 1973. [12] Ф. Рисс, Б. Сёкефальви-Надь, Лекиии по функииональному анализу, Мир, М., 1979. [13] А.Р. Миротин, Матем. заметки, 64:3 (1998), 423-430.

\section{А. Р. Миротин}

Поступило

Гомельский государственный

10.03.2011 университет им. Ф. Скорины E-mail: amirotin@yandex.ru

Исправленный вариант 02.11.2012 